\title{
Tratamento endovascular de pseudoaneurisma de aorta torácica com fístula aorto-brônquica em pós-operatório tardio de cirurgia de correção de coarctação de aorta
}

\section{Endovascular treatment of thoracic aortic pseudoaneurysm with aortobronchial fistula in the late postoperative period of surgical correction of the aortic coarctation}

Ana Augusta Gayoso Neves', Alexandre Gayoso Neves Maia de Oliveira², Roberto Teodoro Beck²,

Ricardo Virginio dos Santos 4 , Francisco Carlos Padilha Moreira 5 , Alexandre Campos Moraes Amato

\begin{abstract}
Resumo
Fístula aorto-brônquica é uma conexão entre a aorta e o brônquio, e mesmo quando imediatamente reconhecida e tratada possui alto risco de letalidade. Pode se desenvolver após cirurgias de aorta, e é geralmente uma consequência de pseudoaneurisma. A hemoptise, massiva ou intermitente, é o principal sintoma apresentado. O tratamento convencional da fístula aorto-brônquica é a cirurgia aberta de aorta torácica, com reconstrução traqueobrônquica. Recentemente, o reparo endovascular tem sido proposto como uma alternativa. Os autores apresentam um relato de tratamento endovascular, realizado com êxito, de pseudoaneurisma de aorta torácica com fístula aorto-brônquica 22 anos após cirurgia para correção de coarctação aórtica.
\end{abstract}

Palavras-chave: Aorta; cardiopatias congênitas; fistula; hemoptise; falso aneurisma.

\begin{abstract}
Aortobronchial fistula is an abnormal passage between the aorta and the bronchus, and even when recognized and treated promptly, it carries a high risk of fatality. It can develop after aortic operations, and it is usually the result of a pseudoaneurysm. Massive or intermittent hemoptysis is the main symptom. Conventional treatment of aortobronchial fistula is open surgery of the thoracic aorta with tracheobronchial reconstruction. Recently, endovascular repair has been proposed as an alternative. The authors report a case of successful endovascular treatment of thoracic aortic pseudoaneurysm with aortobronchial fistula, 22 years after surgical correction of the aortic coarctation.
\end{abstract}

Keywords: Aorta; heart defects, congenital; fistula; hemoptysis; aneurysm, false.

\section{Introdução}

Fístula aorto-brônquica é uma conexão entre a aorta e o brônquio, que pode causar hemoptise severa e fatal. A mortalidade é alta por causa da hemoptise maciça e pela dificuldade do diagnóstico, pois apenas em raríssimos casos a fístula pode ser visualizada em exames de imagem ${ }^{1,2}$. Ela pode se desenvolver a partir de um aneurisma da aorta torácica descendente, após trauma, ou pode surgir como sequela cirúrgica no reparo de defeitos cardíacos congênitos $^{3}$. Mesmo quando imediatamente reconhecida e tratada, a fístula aorto-brônquica possui alto risco de letalidade. Algumas alternativas de tratamento vêm sendo relatadas recentemente, incluindo ponte extra-anatômica e homoenxerto criopreservado, porém pouco sabe-se sobre o tema devido a sua baixa frequência ${ }^{4}$. Os autores apresentam um relato de caso de tratamento endovascular, realizado com êxito, de pseudoaneurisma de aorta torácica com fístula

Trabalho realizado na Universidade de Santo Amaro (Unisa), São Paulo (SP), Brasil.

${ }^{1}$ Acadêmica do $4^{\circ}$ ano de Medicina da Universidade de Santo Amaro (Unisa), São Paulo (SP), Brasil.

${ }^{2}$ Acadêmico do $4^{\circ}$ ano de Medicina da Universidade da Região de Joinville (Univille), Joinville (SC), Brasil.

${ }^{3}$ Chefe serviço de Cirurgia Vascular do Hospital Dona Helena em Joinville, SC. Titular da Sociedade Brasileira de Angiologia e Cirurgia Vascular.

${ }^{4}$ Professor da Disciplina de Cirurgia Vascular da Unisa, São Paulo (SP), Brasil.

${ }^{5}$ Cirurgião Vascular do Hospital Dona Helena em Joinville, SC.

${ }^{6}$ Professor da Disciplina de Cirurgia Vascular da Unisa, São Paulo (SP), Brasil.

Submetido em: 07.09.10. Aceito em: 21.01.11

J Vasc Bras. 2011;10(1):64-67. 
aorto-brônquica em pós-operatório tardio de cirurgia para correção de coarctação aórtica.

\section{Relato de caso}

Paciente do sexo feminino, 38 anos, hipertensa de longa data. Aos 16 anos de idade, realizou cirurgia para correção de coarctação de aorta, por meio da colocação de uma ponte extra-anatômica com enxerto sintético de Dacron. A ponte tinha como anastomose proximal, látero-terminal, a porção justo-subclávia esquerda; distalmente, a anastomose era do tipo término-lateral e encontrava-se no terço médio da aorta descendente.

Vinte e dois anos após a primeira cirurgia, a paciente apresentou durante dois dias uma intensa dor precordial que irradiava para o dorso e era aliviada com o repouso. A partir desse episódio, passou a ouvir um som de rouquidão durante a inspiração, que se agravava com o decúbito dorsal. Três meses depois, deu entrada no pronto-socorro com intensa dor precordial irradiada para o dorso, dispneia grave, hipotensão e tosse acompanhada de hemoptise de grande volume. A paciente nega antecedente de trauma.

Durante a internação, a imagem da tomografia computadorizada evidenciou presença de pseudoaneurisma de aorta (Figura 1). A fístula aorto-brônquica não foi visualizada no exame, porém a história de cirurgia de correção de coarctação da aorta há 22 anos, a presença de pseudoaneurisma, a dor precordial e a hemoptise maciça sugeriram presença de fístula aorto-brônquica tamponada ${ }^{2}$. Foram descartados os diagnósticos de tromboembolismo pulmonar, pneumonia e abscesso pulmonar, sendo indicado o tratamento endovascular de urgência.

Foi feita exclusão endovascular de pseudoaneurisma em aorta torácica, localizada na porção justo-subclávia esquerda até o terço distal da aorta torácica, nível de T10, com colocação de duas endopróteses, Zenith TX2 Z TEG 28-28-140 $\mathrm{mm}$ e 28-24-115 mm (Cook Incorporated, Bloomington,

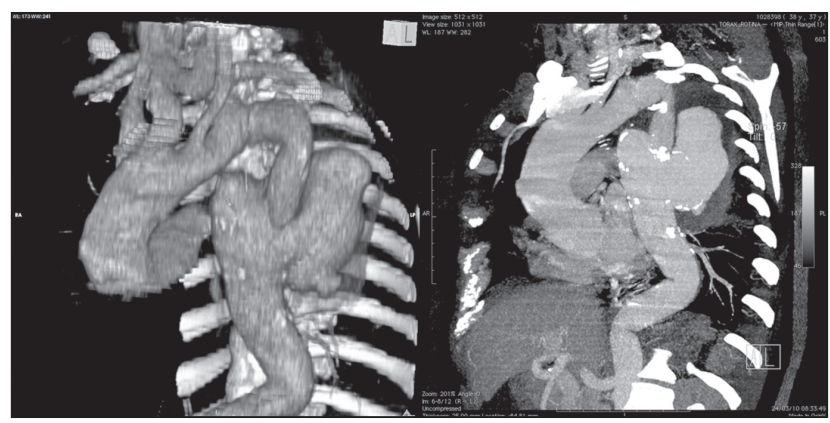

Figura 1 - Reconstrução tridimensional por volume e reconstrução multiplanar evidenciando grande pseudoaneurisma em aorta torácica, com extravasamento de contraste principalmente em anastomose distal
Ind), com telescopagem por via retrógrada pela artéria femoral comum direita. Utilizou-se a técnica do "varal", com um fio-guia 0,14 " introduzido pela artéria braquial direita e exteriorizado na artéria femoral comum direita, devido à dificuldade técnica de cateterismo primário via retrógrada, mantendo assim a estabilidade e a firmeza necessárias para sustentar a endoprótese. O controle angiográfico confirmou aorta pérvia em toda a sua extensão com exclusão do pseudoaneurisma, sem a presença de vazamentos (Figura 2).

No retorno, após três meses, a paciente trouxe o resultado da angiotomografia computadorizada de aorta torácica, mostrando endoprótese pérvia, sem sinais de extravasamento de contraste. Notou-se pequena dissecção focal de aorta abdominal ligeiramente proximal em relação à emergência do tronco celíaco, com deslocamento de flap intimal a partir da parede posterior da aorta, sem trombos (Figuras 3 e 4). Tronco celíaco, artérias mesentérica superior e renais pérvias sem estenoses significativas ou dilatações aneurismáticas evidentes. Paciente se apresentou em bom estado geral, sem queixas, negando dor abdominal, perda de peso ou alteração no hábito intestinal.

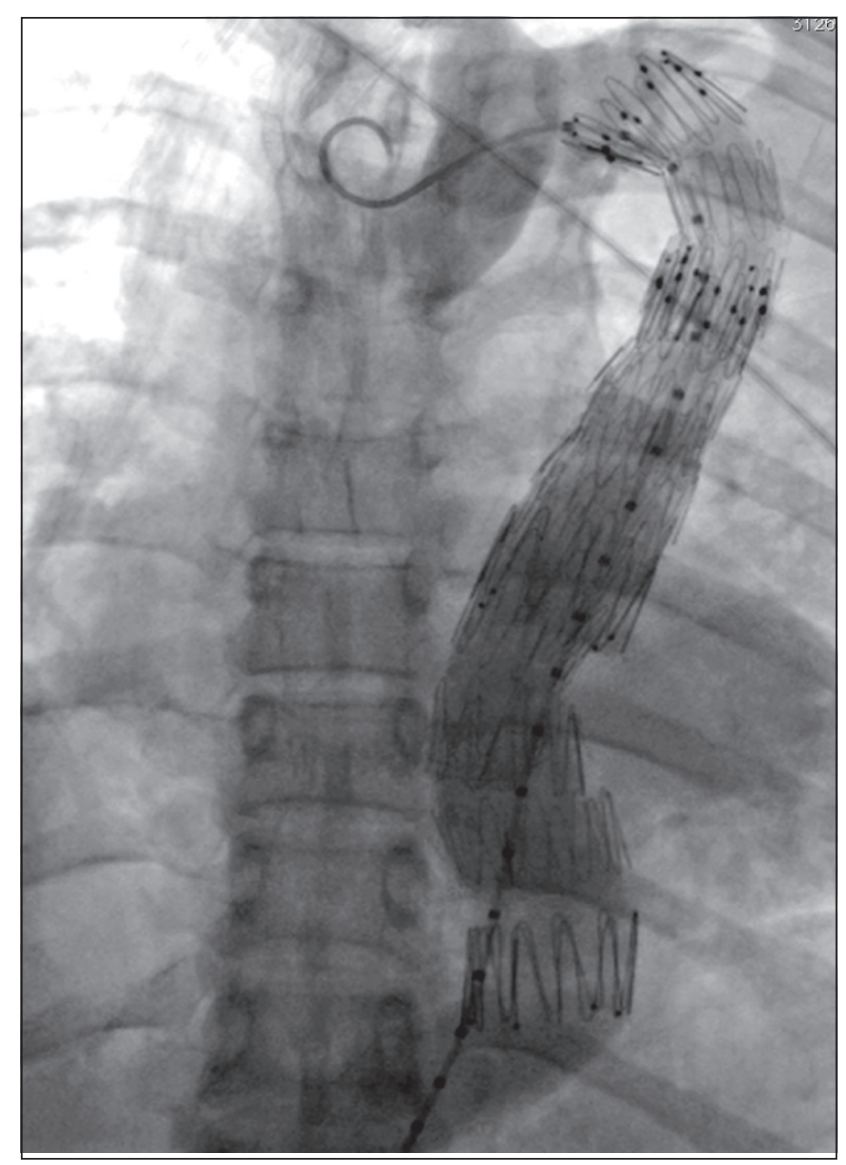

Figura 2 - Arteriografia intraoperatória após liberação das duas endopróteses, demonstrando perviedade e ausência de vazamentos 


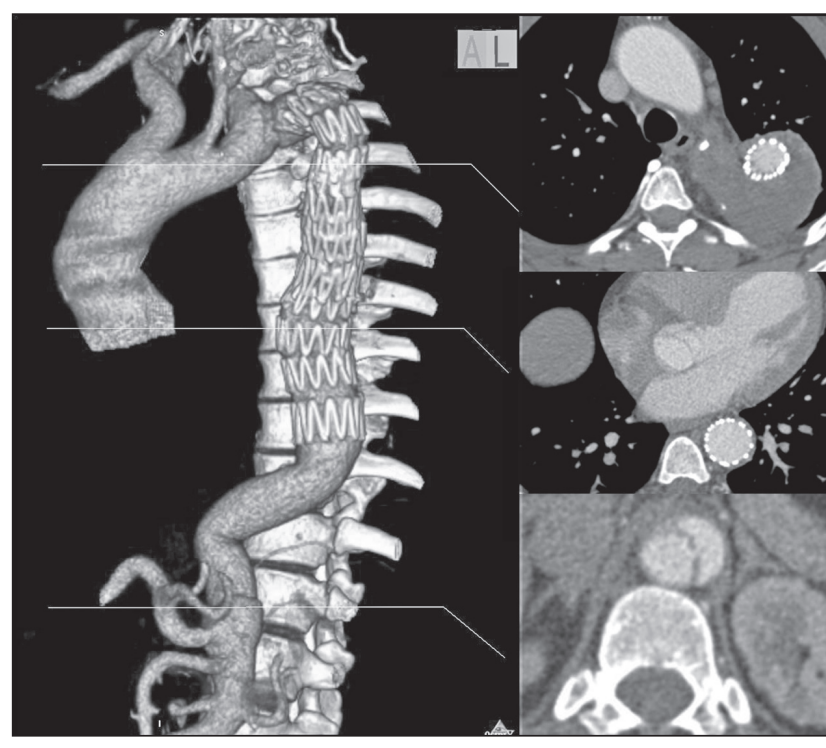

Figura 3 - Reconstrução tridimensional por volume, e imagens axiaischaves de angiotomografia de controle: perviedade das endopróteses e ausência de vazamentos. Imagem compatível com dissecção focal de aorta abdominal ligeiramente proximal em relação à emergência do tronco celíaco, com deslocamento de flap intimal a partir da parede posterior da aorta, sem trombos

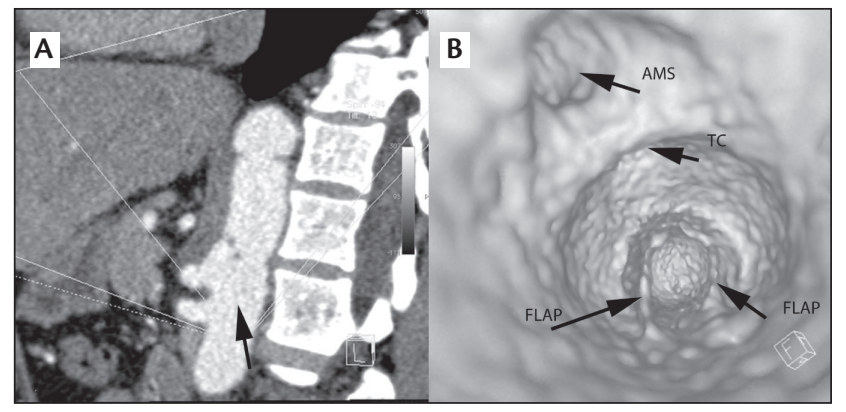

Figura 4 - (A) Seta evidencia, na reconstrução multiplanar, o posicionamento da câmera na reconstrução da imagem B. (B) Reconstrução tridimensional da visão interna do flap intimal, evidenciando perviedade dos óstios da artéria mesentérica superior (AMS) e tronco celíaco (TC)

\section{Discussão}

Fístulas aorto-brônquicas podem se desenvolver em um período indeterminado após cirurgias de aorta, e são geralmente consequência de pseudoaneurisma. A hemoptise, massiva ou intermitente, é o principal sintoma apresentado $^{5,6}$. Diferentes modalidades de diagnóstico podem visualizar o trajeto de fístula aorto-brônquica, porém apenas em raríssimos casos essa visualização é alcançada ${ }^{2}$, como no presente caso, no qual a fístula não foi visualizada na tomografia computadorizada. Hemoptise é um sinal que está presente em inúmeras doenças. Por essa razão, a anamnese é extremamente importante para realizar o diagnóstico diferencial. Em vigência de hemoptise, a presença de cirurgia aórtica prévia é altamente sugestiva de presença de fístula aorto-brônquica, especialmente quando acompanhada de pseudoaneurisma².

O tratamento convencional da fístula aorto-brônquica é a cirurgia aberta de aorta torácica com reconstrução traqueobrônquica. Apesar de algumas estratégias alternativas terem sido reportadas na literatura, incluindo uso de ponte extra-anatômica e correção por meio de homoenxerto criopreservado, não existe um consenso que indique o melhor tipo de tratamento. A taxa de mortalidade da cirurgia aberta está relacionada com as complicações de hemorragia e sepse, as quais chegam a 78\%. O reparo endovascular de aorta torácica tem sido proposto como uma alternativa, pois é um procedimento menos invasivo. Entretanto, a técnica endovascular possui limitações no tratamento da fístula aorto-brônquica, principalmente pelo alto risco de contaminação da prótese ${ }^{4}$.

O tratamento de aorta torácica com próteses endovasculares reduziu significativamente a morbidade associada à toracotomia e ao clampeamento de aorta e reduziu também a mortalidade intra-hospitalar e a morbidade pós-operatória; recentes estudos mostram resultados animadores no médio $\mathrm{prazo}^{7}$. Usando como analogia os casos de pseudoaneurisma de aorta torácica por trauma, nos quais a cirurgia aberta permanece com altas taxas de mortalidade, os recentes relatos de sucesso colocam a técnica endovascular como possível substituta ao tratamento convencional ${ }^{8}$. Apesar do emprego de técnicas endovasculares, a taxa de isquemia da medula espinhal, que tem como consequência paraparesia e/ou paraplegia, mantém-se inalterada quando comparada à cirurgia aberta ${ }^{7}$.

Pouco se sabe sobre os resultados no pós-operatório tardio do uso de endopróteses no tratamento de fístulas aorto-brônquicas. Isso se deve à baixa incidência, à introdução relativamente recente das técnicas endovasculares e à escassez de estudos sobre o tema na literatura ${ }^{4}$.

\section{Conclusão}

O relato mostra a viabilidade da técnica endovascular no tratamento de pseudoaneurisma da aorta torácica com fístula aorto-brônquica, em pós-operatório tardio de cirurgia de correção da coarctação de aorta. 


\section{Referências}

1. YooJH, Lee CT, Shim YS, Chung JW, Ahn H, Kim KW. Aortobronchial fistula presenting as recurrent hemoptysis and successfully treated with an endovascular stent graft. Respiration. 2001;68:537-9.

2. Picichè M, Paulis R, Fabbri A, Chiariello L. Postoperative aortic fistulas into the airways: etiology, pathogenesis, presentation, diagnosis, and management. Ann Thorac Surg. 2003;75:1998-2006.

3. Algaba Calderón A, Jara Chinarro B, Abad Fernández A, Isidoro Navarrete O, Ramos Martos A, Juretschke Moragues MA. Recurrent hemoptysis secondary to an aortobronchial fistula. Arch Bronconeumol. 2005;41(6):352-4.

4. Chiesa R, Melissano G, Marone EM, Kahlberg A, MarroccoTrischitta MM, Tshomba Y. Endovascular treatment of aortoesophageal and aortobronchial fistulae. J Vasc Surg. 2010;51(5): 1195-202.

5. Maclntosh EL, Parrot JCW, Unruh HW. Fistulas between the aorta and tracheobronchial tree. Ann Thorac Surg. 1991;51:515-9.

6. Conlan AA, Hurwitz SS, Krige L, Nicolaou N, Pool R. Massive hemoptysis. Review of 123 cases. I Thorac Cardiovasc Surg. 1983;85:120-4.
7. Chiesa R, Melissano G, Bertoglio L, Campos Moraes Amato A, Tshomba Y, Civilini E, et al. The risk of spinal cord ischemia during thoracic aorta endografting. Acta Chir Belg. 2008;108(5):492-502.

8. Vishal B, Jeanne L, Raul C. Current diagnosis and management of blunt traumatic rupture of the thoracic aorta. J Vasc Bras. 2007;6(1):64-73.

Correspondência:
Alexandre Campos Moraes Amato
Av. Juriti, 144 - Moema - São Paulo, SP
Telefone: (11) 5051-0233
E-mail: dr.alexandre@amato.com.br
Contribuições dos autores
Concepção e desenho do estudo: ACMA, AAGN
Análise e interpretação dos dados: ACMA
Coleta de dados: AAGN, FCPM, AGNMO, RTB
Redação do artigo: ACMA, AAGN
Revisão crítica do texto: ACMA, RVS, RTB
Análise estatística: ACMA, AAGN
Responsana filidade geral pelo estudo: ACMA, RVS, RTB
Informações sobre financiamento: N/A
Rodos os autores leram e aprovaram a versão final submetida ao J Vasc Bras.

